\title{
Roles Del Docente En La Formación Dual En El Ecuador
}

\section{Marcel Oswaldo Méndez Mantuano}

Magister en Gestión Ambiental, Coordinador de la carrera de Procesamiento de Alimentos del Instituto Tecnológico Superior Juan Bautista Aguirre, Ecuador

\section{Amanda Yolanda Lozada Valdez}

Ingeniera en Sistemas Computacionales, Docente investigadora de la Unidad Educativa Galo Plaza Lasso, Ecuador

Ángel Raúl Huayamave Rosado

Ingeniero Agrónomo, Docente investigador del Instituto Tecnológico

Superior Juan Bautista Aguirre, Ecuador Narcisa del Carmen Mantuano Holguín

Licenciada en Ciencias de la Educación, Docente investigadora de Unidad Educativa Rosa Herlinda Martillo Magallanes, Ecuador

\section{Resumen}

La dinámica de la economía global, así como las necesidades de las compañías, han provocado un aumentado de la demanda de profesionales que cuenten con conocimientos específicos en áreas productivas dentro de las empresas. La industria busca personas con capacidad para solucionar problemas puntuales, de manera práctica, sencilla y en el menor tiempo posible. Así mismo, el talento humano de las instituciones educativas está representado en primera instancia por los docentes, los mismos que son la fuente más valiosa del conocimiento desde un aspecto social e institucional, ya que son capaces de generar riqueza intelectual en el alumnado, conducir a logros institucionales importantes para el desarrollo de nuevos saberes, y contribuir al perfeccionamiento individual de aquellos educadores que adiestran a los que erigen la cimiente del mundo moderno. El presente trabajo de investigación, trata de abordar las principales actividades que los docentes duales desempeñan en cada una de las etapas de la formación de los estudiantes (fase teórica y fase práctica); ya que es necesario precisar las funciones regulares que cumplen los docentes que se desenvuelven en carreras de modalidad dual en las instituciones públicas de educación superior del Ecuador. El dinamismo propio de esta propuesta educativa, exige a los docentes una constante actualización de conocimientos, desarrollo de una 
enseñanza orientada a la práctica, simbiosis permanente con las empresas del sector, empatía con los estudiantes, y conocimientos flexibles que asistan a la creación de nuevas propuestas a nivel académico y empresarial.

Palabras claves: Formación dual, docente, tecnológica, roles, retos.

\title{
Roles of the Teacher in Dual Training in Ecuador
}

\section{Marcel Oswaldo Méndez Mantuano}

Magister en Gestión Ambiental, Coordinador de la carrera de Procesamiento de Alimentos del Instituto Tecnológico Superior Juan Bautista Aguirre,

Ecuador

\section{Amanda Yolanda Lozada Valdez.}

Ingeniera en Sistemas Computacionales, Docente investigadora de la Unidad

Educativa Galo Plaza Lasso, Ecuador

Ángel Raúl Huayamave Rosado

Ingeniero Agrónomo, Docente investigador del Instituto Tecnológico

Superior Juan Bautista Aguirre, Ecuador

Narcisa del Carmen Mantuano Holguín

Licenciada en Ciencias de la Educación, Docente investigadora de Unidad

Educativa Rosa Herlinda Martillo Magallanes, Ecuado

\begin{abstract}
The dynamics of the global economy, as well as the needs of companies, have led to an increase in the demand for professionals who have specific knowledge in productive areas within companies. The industry is looking for people with the ability to solve specific problems, in a practical, simple and in the shortest possible time. Likewise, the human talent of educational institutions is represented in the first instance by teachers, who are the most valuable source of knowledge from a social and institutional aspect, since they are capable of generating intellectual wealth in the students, leading to institutional achievements important for the development of new knowledge, and contribute to the individual development of those educators who train those who erect the foundation of the modern world. The present research work, tries to address the main activities that dual teachers perform in each of the stages of student training (theoretical phase and practical phase); since it is necessary to specify the regular functions performed by teachers who work in dual-degree courses in public higher education institutions in
\end{abstract}


Ecuador. The dynamism of this educational proposal, requires teachers to constantly update knowledge, development of a practice-oriented teaching, permanent symbiosis with companies in the sector, empathy with students, and flexible knowledge to assist in the creation of new proposals at academic and business level.

Keywords: Dual training, teaching, technology, roles, challenges.

\section{INTRODUCCIÓN}

La educación es el pilar central de las sociedades modernas, pues juegan un papel crítico en la productividad y en el crecimiento económico de un país, y tiene como eje integrador a los docentes, los mismos que son el valor agregado, los cuales aportan en la determinación del éxito o fracaso de un sistema educativo. Por lo tanto, un sistema efectivo es la combinación de varios elementos, entre los cuales se pueden destacar: el currículo, los estándares educativos nacionales, el liderazgo local de las instituciones, la calidad de la educación, la motivación, las expectativas de los docentes, y una eficaz evaluación de todos los elementos anteriormente expuestos. Los docentes siguen siendo indispensables en la formación de las sociedades, por lo que en la actualidad las autoridades educativas ponen una mayor atención al papel que juegan (OCDE, 2011; Ibarra y Bribiescas, 2019).

Actualmente, en varios institutos tecnológicos superiores del Ecuador se desarrollan carreras de nivel técnico y tecnológico superior, las mismas que demandan de docentes capaces de entender las dinámicas relacionadas a este nivel de formación. A nivel de las instituciones de educación superior, existe el interés de incentivar a que los docentes se formen en las áreas técnicas y tecnológicas, independientemente del título de tercer nivel obtenido en la formación universitaria. Este interés surge al entender que la formación de un técnico responde a destrezas y habilidades frente al uso de herramientas, las del tecnólogo están orientadas a imprimir sus aprendizajes y conocimientos a sus actividades laborales comunes, mientras las carreras universitarias están llamadas a generar conocimiento aplicado en diferentes campos (Unzueta, 2011).

Por estas notables diferencias entre los tipos de formación, surge la imperiosa necesidad de poseer docentes con una formación consistente a la realidad de la academia, ya que la formación de técnicos y tecnólogos busca una interacción entre la investigación y su metodología, la cual, se enfoca no solo en la construcción de procesos, sino en la sistematización de la experiencia, donde se indaga y existe una conceptualización; es por ello, que los técnicos y tecnólogos se forman en el análisis de los procesos y en la construcción del desarrollo tecnológico. 
Dentro de la actual oferta educativa a nivel tecnológico en el Ecuador, existen las carreras denominadas duales, las cuales conjugan al mismo tiempo el aprendizaje teórico en aulas, con la práctica en la vida empresarial, donde se toman ejemplos y modelos reales de tareas y responsabilidades laborales. Los estudiantes (aprendices) se preparan para que, desde el inicio de su formación, puedan tomar decisiones y enfrentar desafíos y retos en su diario vivir profesional (Méndez et al., 2017).

También se pueden recalcar que las carreras de carácter dual, tienen como particularidad preponderante que la instrucción teórica y práctica se imparten equitativa y proporcionadamente durante cada ciclo de estudio, esto se hace considerando que la educación tecnológica requiere de un importante componente práctico. Además, esta tiene ventaja competitiva, por el hecho de que es atractiva y novedosa tanto para la comunidad, así como para las empresas del sector (Ekos, 2016; Méndez et al., 2017).

En las carreras de modalidad dual existe un dinamismo propio, el cual, exige de parte de los docentes una interrelación muy diferente a la de aquellos docentes que se desenvuelven en otras modalidades de enseñanza, ya que las carreras que se desarrollan bajo los principios de la práctica, tienen que desarrollar una enseñanza orientada hacia la acción y todas las actividades realizadas deben girar alrededor de ese paradigma (Alemán, 2015). En este sentido, los docentes en la enseñanza dual, desarrollan actividades académicas bajo la premisa de obtener una formación pertinente a las realidades del tejido productivo y adaptada a la ampliación de conocimientos con el nuevo vínculo, entre empresa y las instituciones educativas; por ello, es indispensable que los docentes fomenten la relación con los procesos industriales al que se haya asociado a los estudiantes (Cámaras de Comercio, Ministerio de Educación, Cultura y Deporte y Fondo Social Europeo, 2012).

Adicionalmente, el docente dual, debe realizar sus funciones de acuerdo a los escenarios de aprendizaje de los estudiantes (teórico y práctico). En el componente teórico, las actividades están dadas por dictar clases en el aula y la realización de prácticas en talleres o laboratorios dentro de la institución educativa. Mientras en el componente práctico (desarrollado por los estudiantes dentro de las empresas formadoras), los docentes están encargados de supervisar que los planes académicos propuestos por la institución educativa, sean cumplidos dentro de las empresas; además, deben tutorear los proyectos empresariales que elaboran los estudiantes dentro de su estadía en la misma (este requisito varía según las instituciones educativas), finalmente los docentes deben desarrollar propuestas de innovación tecnológica para la institución, bajo el concepto de pertinencia y factibilidad. 


\section{METODOLOGÍA}

El presente trabajo de investigación, analizará las actividades que deben cumplir los docentes que colaboran en carreras de formación dual, ya que la dinámica de este tipo de programas, difieren en varios aspectos a los desarrollados en otras modalidades de estudio; con el objetivo de contribuir a la planificación estratégica de las autoridades y los docentes en las instituciones educativas que desarrollen este tipo de enseñanzas. Se aplicó el método cualitativo para describir las actividades de los docentes duales en el componente teórico, las cuales están basados en los articulados legales que rigen la educación superior en el Ecuador. El tipo de investigación es explicativa, ya que se realizan aproximaciones conceptuales, que proporcionan los fundamentos necesarios para modular las actividades que les corresponde desarrollar a los docentes en el componente práctico.

\section{DESARROLLO \\ Roles generales}

En la actualidad la oferta para carreras tecnológicas es realizada por los institutos tecnológicos superiores (ITS) del país, estas competencias fueron otorgadas en la Ley Orgánica de Educación Superior (LOES), cuyo artículo 118 menciona que:

"Art. 118.- Niveles de formación de la educación superior. - Los niveles de formación que imparten las instituciones del Sistema de Educación Superior son:

a) Nivel técnico o tecnológico superior, orientado al desarrollo de las habilidades y destrezas que permitan al estudiante potenciar el saber hacer. Corresponden a éste los títulos profesionales de técnico o tecnólogo superior, que otorguen los institutos superiores técnicos, tecnológicos, pedagógicos, de artes y los conservatorios superiores (...)"

Motivo por el cual, el desarrollo posterior de los enunciados será tomando como referencias los postulados legales dirigidos a los ITS.

De manera general, los docentes en el Ecuador deben cumplir con los requisitos expresados en la Ley Orgánica de Educación Superior (LOES), Reglamento de Carrera y Escalafón del Profesor e Investigador del Sistema de Educación Superior, Reglamento de Régimen Académico y la Normativa para Carreras y Programas en Modalidad Dual.

Entre las principales responsabilidades dadas a los docentes en los articulados legales anteriormente mencionados, tenemos de manera resumida los siguientes:

- Impartición de clases presenciales, virtuales o en línea, en la institución o fuera de ella, incluyendo docencia en servicio y en empresas en la formación dual 
- Formación permanente, asistencia a eventos de capacitación y actualización de conocimientos

- Planificación de sus actividades académicas

- Elaboración de material didáctico y guías académicas

- Planificación de trabajos y prácticas de los estudiantes

- Elaboración de syllabus

- Elaboración y participación de programas o proyectos de vinculación con la sociedad

- Elaboración del portafolio docente (rúbricas de evaluación, planes de clases, planes de estudios, nómina de estudiantes, syllabus, horarios de clases)

- Aplicación y evaluación de exámenes

- Elaboración de reactivos (detalle de preguntas a contestar por el estudiante en una determinada evaluación) para exámenes finales

- Orientación y acompañamiento a través de tutorías presenciales o virtuales, individuales o grupales, tanto a actividades teóricas como prácticas, incluyendo las prácticas pre profesionales y actividades de vinculación con la sociedad

- Dirección y tutorías de trabajos para la obtención del título de Tecnólogo Superior

- Supervisar el estudiante durante su estadía en la empresa formadora y en el desarrollo del proyecto organizacional durante su fase práctica

- Entregar actas de calificaciones del examen final, mejoramiento y rehabilitación del componente teórico y práctico a coordinación de la carrera

- Presentar informes académicos después de cada período, en donde deberá indicar los porcentajes de cumplimiento del programa, los problemas encontrados al impartir el conocimiento a los estudiantes, recomendaciones y cualquier tipo de información correspondiente con el área académica

- Elaboración de informes de gestión académica (detalle de actividades docentes y administrativas realizadas durante un período académico determinado)

- Buscar acercamientos con organizaciones, instituciones y empresas del sector público o privado para la realización de visitas de campo referente a la carrera para permitir a los estudiantes un acercamiento con el sector y la complementación de conocimientos y saberes con hechos y observaciones

- Participación de talleres/reuniones de planificación y/o fortalecimiento institucional 
- Conocimiento y cumplimiento de Leyes, Reglamentos que regulan el quehacer académico

Los docentes que ingresan al sistema de educación superior, deben poseer previamente una trayectoria académica y experiencia práctica en el sector correspondiente a la carrera objeto, permitiéndole contribuir con aprendizajes integrales, significativos y sentar las bases para incorporar estos aprendizajes en la práctica en una empresa específica y en el sector en general. Aparte de los requisitos legales del Reglamento de Carrera y Escalafón del Profesor e Investigador del Sistema de Educación Superior (principalmente), el docente para las carreras tecnológicas bajo la modalidad dual, debe contar con experiencia empresarial de por lo menos dos años en el área afín a la asignatura.

Existen responsabilidades adicionales que los docentes duales deben realizar, las mismas que no nacen de leyes o normativas, sino de la experiencia en el desarrollo de este tipo de carreras, entre las que podemos resaltar las siguientes:

- Fomenta el desarrollo de conocimientos, habilidades, destrezas, actitudes y valores asociados al razonamiento lógico, crítico y creativo

- Demuestra actitud positiva hacia el conocimiento de las disciplinas objeto de cada uno de los ciclos de estudio

- Integra contenidos de las diversas disciplinas contempladas en los diferentes ciclos de estudio, de manera coherente con los objetivos de cada uno de ellos

- Desarrolla modelos conceptuales y relativos a los temas disciplinares, que guíen el aprendizaje orientado hacia la formación integral del futuro técnico y tecnólogo

- Facilita el logro de aprendizajes

- Desarrolla estrategias pedagógicas para que el estudiante identifique sus propias necesidades de aprendizaje, orienta la búsqueda de información pertinente y actualizada y desarrolle competencias de investigación, reflexión y análisis de su realidad.

- Organiza sus clases haciendo uso de didácticas y técnicas de aprendizaje, centradas en el que aprende, desarrollando contextos pedagógicos interactivos y de construcción innovadora del conocimiento y los saberes, desde un enfoque de diálogo de saberes

- Crea ambientes positivos, de respeto a la diversidad cultural, generacional, física, socioeconómica, etc. para estimular la planificación de los diferentes aprendizajes previstos en cada período

- Demuestra compromiso con el proceso formativo y aplica los principios de la bioética, interculturalidad, género, ambiente y 
discapacidad en cada una de sus planificaciones y actuaciones docentes

- Se integra, interactúa y trabaja en equipo con los demás actores docentes y administrativos del proceso formativo

- Es capaz de transmitir a los estudiantes sus experiencias vividas y tecnologías en el ejercicio de la práctica profesional

- Es capaz de entablar diálogos amistosos con los estudiantes y los actores relacionados con el sector en un contexto de respeto a la diversidad

Cabe mencionar que la asignación de la "carga horaria" se encuentra entre 18 y 22 horas destinadas para dictar clases (docentes a tiempo completo), y las restantes horas laborales (o complementarias) comprenden a las actividades anteriormente descritas y varían según los docentes (depende del área institucional en la que se encuentre). Es decir, las horas complementarias en un determinado período academico (la distribución se la realiza por período, existen 2 por año), no se modifican; la variación de las actividades se encuentran exclusivamente en las horas asignadas para clases en la fase teórica, que posteriormente se transforman en "actividades duales" en la fase práctica (paralelamente a las actividades de los estudiantes dentro de las empresas).

\section{Rol del docente en el componente teórico}

Como se lo mencionó anteriormente, las actividades docentes en un determinado período académico, están seccionadas en dos fases o componentes. La fase teórica y la fase práctica; cada una tiene un peso similar en las horas de aplicación, sin embargo, no es una regla estándar, ya que existen diseños curriculares que le dan un diferenciado porcentaje a cada fase (depende de la estructura de la carrera y del tejido empresarial en la que se desarrolla). De manera general existe un consenso para determinar un porcentaje de horas similares a la fase teórica y práctica, el cual es del $50 \%$ en cada uno.

Los docentes duales imparten sus clases teóricas y de experimentación práctica (dentro de talleres y laboratorios), en un lapso similar al número de semanas que los estudiantes se encuentran en las "empresas formadoras" (denominación para especificar aquellas empresas que acogen estudiantes en modalidad dual).

En las semanas que conforman la fase teórica, los docentes dictan las cátedras correspondientes a cada una de las asignaturas, siguiendo la organización curricular acorde con el nivel de aprendizaje en cada período académico, y contrastando los resultados de aprendizajes con el plan de rotación; este último es el instrumento de gestión y control individual del proceso de formación práctica de los estudiantes que se encuentran en una 
determinada empresa formadora, tiene como propósito establecer las tareas, funciones y actividades que el estudiante realiza semana a semana, de acuerdo a un cronograma de ejecución. En caso de ser necesario, se acoplan nuevos contenidos o metodologías que ayuden al desarrollo del plan de rotación en la fase práctica.

La correcta impartición de los conocimientos en los estudiantes, permite la comprensión y contextualización de las problemáticas del área de conocimiento de su carrera, logrando una articulación entre la teoría aprendida en el instituto y la práctica desarrollada en las empresas formadoras.

Los docentes deben desarrollar el aprendizaje con los estudiantes bajo cuatro actividades:

- Actividades de aprendizajes asistidos, las cuales corresponden a aquellas actividades que se realizan con el acompañamiento del docente y el estudiante en los diferentes ambientes de aprendizaje, por ejemplo: clases presenciales, conferencias, seminarios, orientación para estudio de casos, foros, docencia realizada en escenarios laborales, entre otras

- Actividades de aprendizaje colaborativo, el cual es el trabajo en grupo de los estudiantes con interacción permanente del docente. Entiéndase como actividades de aprendizaje colaborativo a los proyectos de integración de saberes, la construcción de modelos y prototipos, proyectos de problematización y resolución de problemas o casos, la sistematización de prácticas de investigación e intervención; o todas las actividades que apliquen metodologías de aprendizaje que fomenten el uso de diversas tecnologías de la información y la comunicación, así como también metodologías en red, tutorías in situ o en entornos virtuales

- Actividades de prácticas de aplicación y experimentación de los aprendizajes, se refiere a actividades académicas desarrolladas en escenarios experimentales o en laboratorios. Entre estas podemos citar: prácticas de campo, trabajos de observación dirigida, resolución de problemas, talleres, manejo de base de datos y acervos bibliográficos

- Actividades de aprendizaje autónomo, comprende el aprendizaje independiente e individual del aprendizaje. Este trabajo será diseñado, planificado y orientado por los docentes, para alcanzar los objetivos y el perfil de egreso de la carrera o programa. Algunas de las actividades que se contemplan en este componente son: lectura crítica, análisis y comprensión de materiales bibliográficos y documentales, generación de datos, búsqueda de información, elaboración individual de ensayos, trabajos, exposiciones, etc.

Las carreras de carácter práctico asocian la investigación experimental durante el proceso de aprendizaje, por ello, el docente debe construir durante 
el mismo, las herramientas cognitivas que permitan a los estudiantes asimilar un rol de investigadores, ya que deben establecer sin dificultad las relaciones causa-efecto que se producen en su entorno, para tratar de dar solución a las necesidades que surgen en el componente práctico.

Durante cada fase teórica, el docente en las materias afines a la práctica o experimentación, debe realizar al menos una visita de campo, para permitir a los estudiantes un acercamiento al sector y la complementación de conocimientos y saberes con hechos y observaciones.

\section{Rol del docente en el componente práctico}

Lo expresado a continuación no está respaldado por ninguna normativa o reglamentación legal que relacione la labor docente en la formación dual, ya que existe un vacío legal que justifique las acciones a enunciar. Este mismo vacío ocasiona que los administradores del talento humano en la educación superior del Ecuador, consideren la realización de una "fiscalización" (en algunos casos) de estas horas no ejecutadas como "horas de clases"; esto se subsanaría, con el entendimiento que el rol de los docentes duales evoluciona en la segunda etapa de los períodos académicos (fase práctica), de tal manera, que las horas no ejecutadas como clases, son ahora actividades académicas (duales), que pueden ser justificadas y monitoreadas, de manera constante o con los resultados previstos. En este sentido, es indispensable proponer a las autoridades respectivas, el diseño de una normativa especial que permita justificar la propuesta metodológica dual, ya que se corre el riesgo que los ITS modelen (de tal manera que se pierda la esencia de la dualidad) o excluyan dentro de su ofertas académicas las carreras duales.

Una vez finalizada la fase teórica, de manera automática los roles de los docentes se transforman en "actividades duales". Entre las que comprenden:

- Supervisión del plan de rotación: consisten en comprobar in situ que las actividades que realizar los estudiantes durante la fase práctica, sean las establecidas por el plan de rotación, de ser necesario, se deben realizar los ajustes respectivos para que exista coherencia entre este y los resultados de aprendizajes

- Tutorear los proyectos empresariales: esta actividad es realizada de manera paralela a las actividades que ejecutan los estudiantes dentro de las empresas formadoras, por ello, se asignan horas (en promedio 4 horas semanales por proyecto) para realizar el análisis de pertinencia de los proyectos que se están desarrollando. El docente tutor dual, debe permitir que, durante la elaboración del proyecto, este interactúe de manera armónica entre los conceptos teóricos aprendidos en la institución educativa y situaciones reales presentes en las empresas; y con ello, lograr una simbiosis que dé como resultado un proyecto que 
aporte académica y empresarialmente, pasando por el estímulo de el "saber", el "saber hacer" y el "saber ser", es decir, lo conceptual, lo procedimental y lo actitudinal

- Desarrollo propuestas de innovación tecnológica para la institución: uno de los ejes principales de la academia, es que los docentes sean agentes de producción del conocimiento científico, y para cumplir con esta expectativa, los docentes duales destinan horas a la investigación en las áreas afines de las carreras, cuyos resultados son los artículos científicos presentados, ponencias, seminarios o cursos dados, realización de productos nuevos, desarrollo de patentes, entre otras

\section{CONCLUSIÓN}

Los docentes duales realizan sus funciones de acuerdo a dos escenarios de aprendizaje de los estudiantes: la fase teórica y la fase práctica.

En el componente teórico, las actividades están dadas por la impartición de clases y la realización de prácticas en talleres o laboratorios en la institución educativa.

En el componente práctico, los docentes supervisan el cumplimiento del plan de rotación, realizan tutorías de los proyectos empresariales que elaboran los estudiantes y plantean propuestas de innovación tecnológica para la institución, que son evidenciadas mediante artículos, patentes, seminarios, cursos, etc.

Es imperiosa la realización de una normativa exclusiva para los docentes en modalidad dual, ya que no existen lineamientos específicos que determinen las actividades durante la ejecución del componente práctico.

\section{References:}

1. Alemán, J. (2015). El sistema dual de formación profesional alemán: escuela y empresa. Educação e Pesquisa, 41 (2), 495-511.

2. Cámaras de Comercio, Ministerio de Educación, Cultura y Deporte y Fondo Social Europeo. (2012). Guía de formación dual [archivo PDF]. España: Cámaras de Comercio, Ministerio de Educación, Cultura y Deporte y Fondo Social Europeo. Recuperado de http://www.cnse.es/guia_formacion_dual/pricipal/pdf/GuiaFormacion Dual.pdf.

3. Ekos. (2016). Sistema de Formación Dual. Un cambio de paradigma que conecta la teoría y la práctica profesional. Ekos Negocios, 265, 5859.

4. Ibarra, M.; Bribiescas, F. (2019). Educacion Dual: Su Análisis Y Desarrollo Del Modelo Alemán Para Su Implementacion En El Entorno Laboral. European Scientific Journal, 15 (4), 143-157. DOI: http://dx.doi.org/10.19044/esj.2019.v15n4p143. 
5. Ley Orgánica de Educación Superior, LOES. Registro Oficial Suplemento 298, Quito, Ecuador, 12 de octubre del 2010.

6. Méndez, M.; Campi, I.; Huayamave, R.; Lozada, A. (2017). Modelo de formación dual en el Instituto Tecnológico Superior "Juan Bautista Aguirre". Pontificia Universidad Católica del Ecuador. Recuperado de http://repositorio.pucesa.edu.ec/bitstream/123456789/2309/1/Modali dad.pdf.

7. Normativa para Carreras y Programas en Modalidad Dual. RPC-SO 31-No.585-2016, Quito, Ecuador, 06 de septiembre del 2016.

8. Reglamento de Carrera y Escalafón del Profesor e Investigador del Sistema de Educación Superior (Codificación). RPC-SO-037-No.Z652012, Quito, Ecuador, 13 de julio del 2017.

9. Reglamento de Régimen Académico, RRA. RPC-SO-17-No.2692016, Quito, Ecuador, 04 de mayo del 2016.

10. OCDE, Organización para la Cooperación y el Desarrollo Económicos. (2011). Establecimiento de un marco para la evaluación e incentivos docentes: Consideraciones para México. México DF, México: OECD Publishing.

11. Unzueta, S. (2011). Educación técnica, tecnológica y productiva para adultos desde una perspectiva neurodidáctica, crítica, reflexiva y propositiva. Revista Integra Educativa, 4 (1), 85-115. 